\title{
Simple Estimates of Polar Amplification in Moist Diffusive Energy Balance Models
}

\author{
Timothy M. MERLis AND MATTHEw HenRy \\ McGill University, Montreal, Quebec, Canada
}

(Manuscript received 28 August 2017, in final form 19 April 2018)

\begin{abstract}
Diffusive energy balance models (EBMs) that use moist static energy, rather than temperature, as the thermodynamic variable to determine the energy transport provide an idealized framework to understand the pattern of radiatively forced surface warming. These models have a polar amplified warming pattern that is quantitatively similar to general circulation model simulations. Even without surface albedo changes or other spatially varying feedbacks, they simulate polar amplification that results from increased poleward energy transport with warming. Here, two estimates for polar amplification are presented that do not require numerical solution of the EBM governing equation. They are evaluated relative to the results of numerical moist EBM solutions. One estimate considers only changes in a moist thermodynamic quantity (assuming that the increase in energy transport results in a spatially uniform change in moist static energy in the warmed climate) and has more polar amplification than the EBM solution. The other estimate uses a new solution of a truncated form of the moist EBM equation, which allows for a temperature change that is consistent with both the dry and latent energy transport changes, as well as radiative changes. The truncated EBM solution provides an estimate for polar amplification that is nearly identical to that of the numerical EBM solution and only depends on the EBM parameters and climatology of temperature. This solution sheds light on the dependence of polar amplification on the climatological temperature distribution and offers an estimate of the residual polar warming in solar radiation management geoengineered climates.
\end{abstract}

\section{Introduction}

The pattern of surface warming from radiative forcing robustly peaks in the Arctic in future projections of Earth's climate and the Southern Hemisphere warming is expected to be amplified in high latitudes in equilibrium climate states. This polar-amplified pattern of warming can arise from multiple factors. A canonical explanation for polar amplification (PA) is that it arises from surface albedo feedback that results from a loss of sea ice or a decrease in snow. However, in comprehensive climate change simulations, there are also substantial changes on the longwave radiation side of the top-of-the-atmosphere (TOA) energy budget in the Arctic (Winton 2006; Pithan and Mauritsen 2014), consistent with an increase in poleward atmosphere-ocean energy transport (Zelinka and Hartmann 2012; Huang and Zhang 2014). Increased convergence of energy transport provides a means of enhanced Arctic warming

Corresponding author: Timothy M. Merlis, timothy.merlis@ mcgill.ca (e.g., Solomon 2006), and this alternate avenue to PA can help explain why climate model simulations that do not include temperature-dependent surface albedo still simulate PA (Alexeev 2003; Hall 2004). Here, we use simple estimates to quantify the PA that arises from increased energy transport under warming with the goal of understanding what controls the PA's order of magnitude in the idealized case that does not include surface albedo changes.

Diffusive energy balance models (EBMs) have provided an important idealized framework to address a variety of climate change questions (Sellers 1969; North 1975; North et al. 1981; Flannery 1984; Frierson et al. 2007; Kang et al. 2009; Hwang et al. 2011; Rose et al. 2014; Merlis 2014; Roe et al. 2015). The typical thermodynamic variable used in these models to determine energy transport is temperature (dry EBMs; e.g., Sellers 1969; North et al. 1981). With this choice, PA can only occur if there is spatial inhomogeneity in the radiative forcing or radiative feedbacks that tends to produce it. Replacing temperature diffusion with moist static energy (MSE) diffusion (moist EBMs) allows for the latent 
component of the energy transport to increase with temperature (Flannery 1984) in a way that is similar to the behavior of general circulation models (GCMs; e.g., Frierson et al. 2007; Caballero and Hanley 2012). Moist diffusive EBMs produce polar-amplified warming even in the absence of spatially varying radiative forcing or feedbacks as the result of the increase in energy transport with warming (Flannery 1984; Rose et al. 2014). These simulations have a factor of 1.5-2.7 more warming at the pole than at the equator (Rose et al. 2014; Roe et al. 2015), which is comparable to comprehensive climate change simulations in the Arctic (Collins et al. 2013). We note that the destabilizing lapse rate change in the Arctic also contributes to enhanced surface warming in both comprehensive and idealized GCM simulations of climate change (Pithan and Mauritsen 2014; Feldl and Roe 2013; Henry and Merlis 2018, manuscript submitted to J. Climate) and that the typical formulation of moist EBMs - including those described here-does not include this lapse rate change (although it can be prescribed through the longwave feedback parameter; e.g., Rose et al. 2014). Also, while diffusive EBMs can capture the downgradient transport of transient extratropical eddies, there may also be an important role for other dynamical mechanisms, like stationary waves, to enhance Arctic warming (Lee 2014).

There is a significant body of research on dry EBM theory; that is, EBM solutions that do not rely on numerical integration of the governing equation (North 1975; North et al. 1981; Rose et al. 2017). In contrast, moist EBMs have only been numerically solved to date. Given the similarity of the pattern of warming to that of substantially more comprehensive models, we believe it is worthwhile to examine simple estimates that attempt to capture the moist EBM behavior without resorting to a numerical solution of the moist EBM governing equation. We provide two simple estimates to capture the effect of an increase in energy transport and a concomitant polar-amplified warming pattern. One simple estimate is that of the temperature change from a spatially uniform increase in MSE with warming [inspired by Byrne and O'Gorman (2013b)]. For the other estimate, we adapt the truncated-spectral solution described by North (1975) for dry EBMs to the case of the moist EBM. In our approach, the addition of moisture has two effects on the truncated solution. First, it is analogous to diffusing temperature, albeit with a spatially inhomogeneous diffusivity, for which we can use existing approaches to solve spectrally truncated versions of the dry EBM (North 1975). Second, it gives rise to the addition of a latent energy flux convergence term for perturbed climates that appears in the solution as a "forcing" term, like absorbed solar radiation.
In the next section, we describe the moist EBM formulation and present the results of numerical solutions (section 2). Then, we present simple estimates (section 3) and compare the changes in the temperature, MSE, and the TOA budget of the estimates to those of the numerical moist EBM solutions. The comparison of the estimates to the EBM results provides physical insight into the origin of the magnitude of the PA in this idealized framework. We therefore discuss both estimates, even though the first of the estimates produces substantially more PA than the EBM and more of the discussion is devoted to our newly formulated spectral truncation of the moist EBM equation that successfully captures the warming pattern of the numerical EBM. We subsequently apply the spectral truncation to a wide range of climates to shed light on how the estimate depends on the climatology of temperature and apply it to obtain a GCM-free estimate of "residual" polar warming in solar radiation management geoengineered climate states (section 4). Last, we discuss the application of the simple estimates to Earth's climate and conclude (section 5).

\section{Moist EBM}

We present the solutions of EBM governed by the following equation:

$$
C \partial_{t} T(\phi)=\frac{1}{4} Q S(\phi) a(\phi)-[A+B T(\phi)]-\nabla \cdot \mathbf{F}_{a}(\phi)+\mathcal{F},
$$

with heat capacity $C$, surface temperature $T$, latitude $\phi$, solar constant $Q=1360 \mathrm{~W} \mathrm{~m}^{-2}$, insolation structure function $S(\phi)$, coalbedo $a(\phi)$, outgoing longwave radiation $A+B T$, atmospheric energy flux divergence $\nabla \cdot \mathbf{F}_{a}$, and radiative forcing $\mathcal{F}$. Here, the divergence of the atmospheric energy flux is governed by diffusion of MSE $h$, $\nabla \cdot \mathbf{F}_{a}=-\partial_{x}\left[\mathcal{D}\left(1-x^{2}\right) \partial_{x} h\right]$, with $x=\sin \phi$ and $\mathcal{D}$ the diffusivity, and MSE defined in units of temperature as $h=T+L \mathcal{H} c_{p}^{-1} q^{*}(T)$. Here, the latent heat of vaporization is constant $L=2.5 \times 10^{6} \mathrm{~J} \mathrm{~kg}^{-1}$, the relative humidity $\mathcal{H}$ is a uniform value of $80 \%$, the heat capacity of air at constant pressure $c_{p}=1004.6 \mathrm{~J} \mathrm{~kg}^{-1} \mathrm{~K}^{-1}$, and potential energy is neglected as all variables are defined at the surface $\left(z=0\right.$ and $\left.p_{s}=10^{5} \mathrm{~Pa}\right)$. We assume a single liquid-to-vapor phase transition with the parameter values of the Clausius-Clapeyron (CC) relation given in O'Gorman and Schneider (2008) to determine the saturation specific humidity $q^{*}(T)$.

For the shortwave radiation, we follow North et al. (1981) and use the insolation structure function $S(x)=$ $1-\gamma P_{2}$, with $\gamma=0.482$ and the second Legendre 
polynomial $P_{2}=1 / 2\left(3 x^{2}-1\right)$. This is a time-independent insolation that is representative of Earth's annual mean. The coalbedo is given by $a(x)=a_{0}+a_{2} P_{2}$, with $a_{0}=0.68$ and $a_{2}=-0.2$ (North et al. 1981). This captures the climatological variations in surface albedo and shortwave cloud radiative effects: the planetary albedo increases toward the pole. However, $a(x)$ does not have any temperature dependence, which eliminates the surface albedo and shortwave cloud radiative feedbacks.

We use a longwave feedback parameter that is spatially uniform with a value of $B=1.8 \mathrm{~W} \mathrm{~m}^{-2} \mathrm{~K}^{-1}$. In section 3 , we consider the case of spatially uniform forcing $\mathcal{F}=3.6 \mathrm{~W} \mathrm{~m}^{-2}$, which is representative of the global-mean radiative forcing of a doubling of $\mathrm{CO}_{2}$. With these parameter values, there is $2 \mathrm{~K}$ of global-mean warming; however, the measure of PA that we use-the ratio of polar-to-equatorial warming-is a normalized one that is independent of the magnitude of the global warming or radiative forcing. In contrast to this idealized EBM configuration, the longwave feedback parameter and radiative forcing of Earth's climate do have spatial structure (Winton 2006; Huang et al. 2016). For Earth's climate the combination of the Planck feedback (increase in TOA radiative flux from a vertically uniform warming) and forcing would produce tropically amplified warming if there were a local radiative balance (Henry and Merlis 2018, manuscript submitted to J. Climate), although the lapse rate, water vapor feedback, and energy transport can modify this tendency (Feldl and Roe 2013; Pithan and Mauritsen 2014). The role of the spatial structure in radiative forcing is discussed more in section $4 \mathrm{~b}$.

The EBM equation is numerically integrated with a second-order finite-difference discretization for the diffusion operator and a fourth-order Runge-Kutta time-stepping scheme [similar to Merlis (2014) and Wagner and Eisenman (2015)] over a domain that spans both hemispheres with 180 grid points spaced uniformly in $x$. All numerical EBM results are integrated to steady states. The value of $\mathcal{D}=0.3 \mathrm{~W} \mathrm{~m}^{-2} \mathrm{~K}^{-1}$ is chosen to give an Earth-like control simulation (with $\mathcal{F}=0 \mathrm{~W} \mathrm{~m}^{-2}$ and $A=-281.67 \mathrm{~W} \mathrm{~m}^{-2}$, a typical value that is equivalent to $A=210 \mathrm{~W} \mathrm{~m}^{-2}$ if the units of the EBM are in degrees Celsius as is conventional) with a global-mean surface temperature of $288.6 \mathrm{~K}$ and an equator-pole difference of $46.5 \mathrm{~K}$. Table 1 has the Legendre polynomial expansion of the control simulation. The diffusivity $\mathcal{D}$ is held fixed when the climate is perturbed, as is conventional in EBM analyses, but it is important to note that this neglects changes in the atmospheric circulation (e.g., extratropical eddy kinetic energy) that should affect it (e.g., Caballero and Hanley 2012; Shaw and Voigt 2016).
TABLE 1. Legendre polynomial expansion of the surface temperature $\left(T=\sum_{n} T_{n} P_{n}\right)$ of the numerical EBM solutions in units of kelvin. The linearized MSE $\tilde{h}$ is defined by (4). The $T_{2}$ coefficient can be converted into an equator-pole temperature difference by multiplying by 1.5 . Higher-order coefficients are smaller than 0.4 .

\begin{tabular}{ccccc}
\hline \hline MSE & $\mathcal{F}\left(\mathrm{W} \mathrm{m}^{-2}\right)$ & $T_{0}$ & $T_{2}$ & $T_{4}$ \\
\hline$h$ & 0 & 288.6 & -29.3 & -4.9 \\
$h$ & 3.6 & 290.6 & -28.0 & -4.8 \\
$\tilde{h}$ & 0 & 288.6 & -29.3 & -4.9 \\
$\tilde{h}$ & 3.6 & 290.6 & -28.0 & -4.8 \\
\hline
\end{tabular}

Given that we use constant coefficient EBM parameter values $(B, \mathcal{F})$, this would imply a spatially uniform warming given by $\mathcal{F} / B$ for dry EBMs $(\mathcal{H}=0)$. This radiative equilibrium temperature change does not have spatial variation that would provoke a change in the energy transport in the dry limit. For moist EBMs, the radiative equilibrium warming has increased MSE gradients that cause a corresponding increase in the energy transport. The divergence of this increased energy transport is a cooling tendency in low latitudes and a warming tendency in high latitudes and therefore leads to a polar amplified warming pattern.

The simulated pattern of temperature change in the moist EBM is shown in Fig. 1a. Consistent with previous results (Flannery 1984; Rose et al. 2014), this configuration results in PA with about 2.3 times the polar warming as the tropical warming in the absence of destabilizing high-latitude feedbacks. This is smaller than the observed Arctic warming in the last half century, which has roughly 3 times as much zonal-mean Arctic warming compared to tropical warming (according to the GISTEMP dataset; Hansen et al. 2010).

As we will consider spectrally truncated solutions in what follows, we also plot the temperature change pattern truncated at the second Legendre polynomial component. We use the notation $P_{n}$ for the $n$th Legendre polynomial and the coefficients are denoted with the corresponding subscript (e.g., $T_{2}$ is the second Legendre polynomial coefficient of surface temperature). The odd $P_{n}$ coefficients are zero because we consider hemispherically symmetric climate states. This spectral truncation-the sum of the change that is $\propto x^{2}=\sin ^{2}(\phi)$ and the global-mean $P_{0}$ component-is quite close to the full temperature change pattern. It differs by a few percent from the full change (Fig. 1a, dashed line), consistent with the smallness of changes in the higherorder components of the spectral expansion (Table 1). Because the second Legendre polynomial component dominates here, the measure of polar amplification we use can be converted to others. Our measure (ratio of poleto-equator temperature change) is about $20 \%$ larger than 

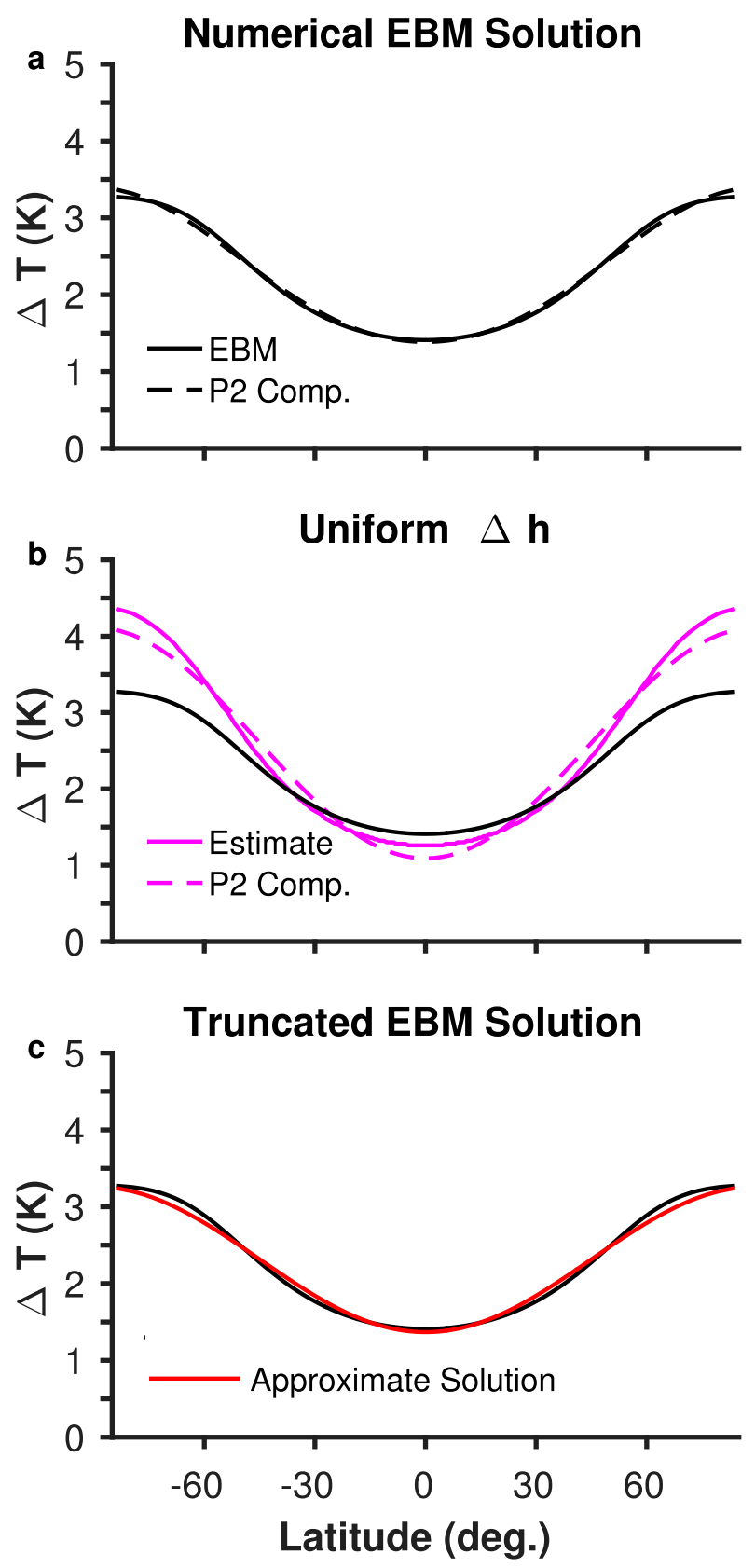

FIG. 1. Change in surface temperature for (a) the numerical EBM solutions, (b) the estimate assuming a uniform increase in MSE (magenta) (2), and (c) the second Legendre polynomial $P_{2}$ truncated solution to the EBM equation in (12) (red). Dashed lines in (a) and (b) show the $P_{2}$ truncation.

the ratio of the more commonly used area average of the temperature change in a polar cap of $60^{\circ}-90^{\circ}$ latitude compared to the tropical belt of $0^{\circ}-30^{\circ}$ latitude for the standard parameter values, although this difference grows larger with increased PA.

Figure 2a shows the simulated pattern of MSE change in the moist EBM (black) together with the MSE change
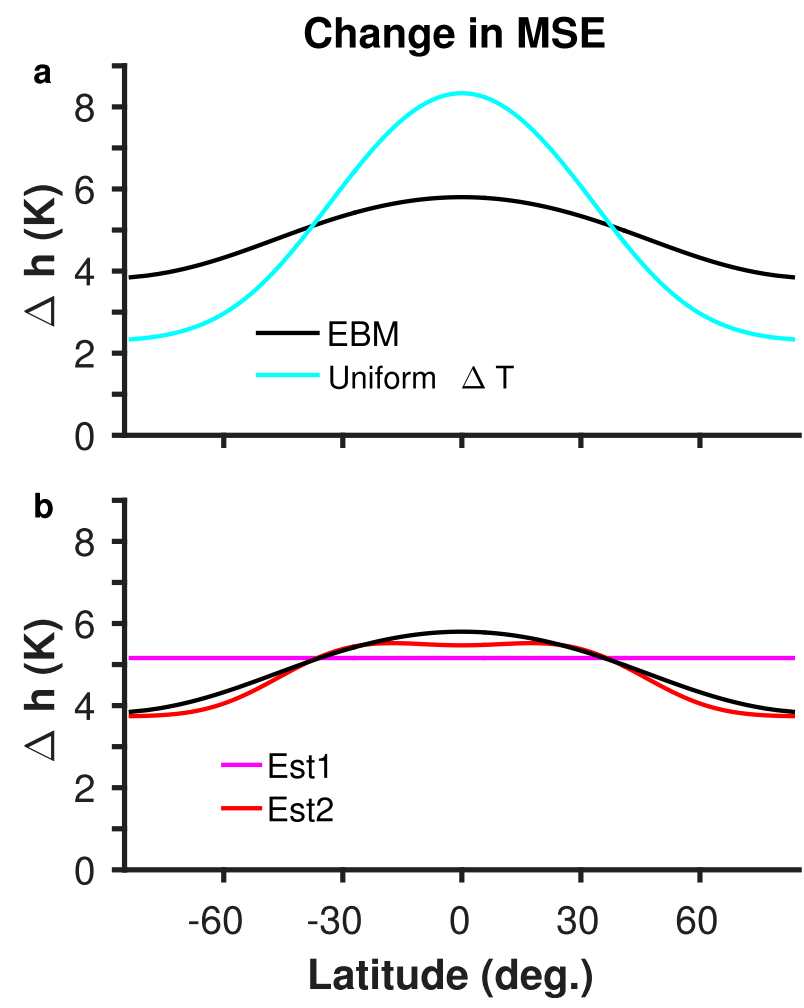

FIG. 2. (a) Change in MSE for the numerical EBM solutions (black) and uniform warming (cyan). (b) Change in MSE for the estimate assuming a uniform increase in MSE from (2) (magenta) and the truncated solution to the EBM equation in (12) (red).

of uniform, radiative warming of $\mathcal{F} / B$ with constant relative humidity (cyan). The moist EBM has increased equator-pole MSE gradients in the warmed climate: approximately $0.6-\mathrm{K}$ larger increase in low latitudes compared to the global-mean increase of $5.2 \mathrm{~K}$ and approximately $1.3-\mathrm{K}$ smaller increase in high latitudes. However, consistent with the discussion above, this perturbation MSE gradient in the EBM simulations is about half that of the uniform warming. The downgradient diffusion has partially homogenized the perturbation MSE.

Figure 3a shows components of the perturbation TOA energy budget. The change in the outgoing longwave radiation (OLR), $B \Delta T-\mathcal{F}$, is shown in the solid line and the change in MSE flux convergence, $-\Delta\left(\nabla \cdot \mathbf{F}_{a}\right)$, is shown in the gray dashed line. Given that we show equilibrated simulations $\left(\partial_{t} T=0\right)$ with unchanged absorbed solar radiation, the two lines fall on top of each other: from (1), $B \Delta T-\mathcal{F}=-\Delta\left(\nabla \cdot \mathbf{F}_{a}\right)$. The high-latitude MSE flux convergence has increased with warming and the enhanced polar warming gives rise to a corresponding increase in local OLR. In the simple estimates presented in what follows, there can be discrepancies between these terms in the energy budget, which can shed light on the limitations of the estimates. 

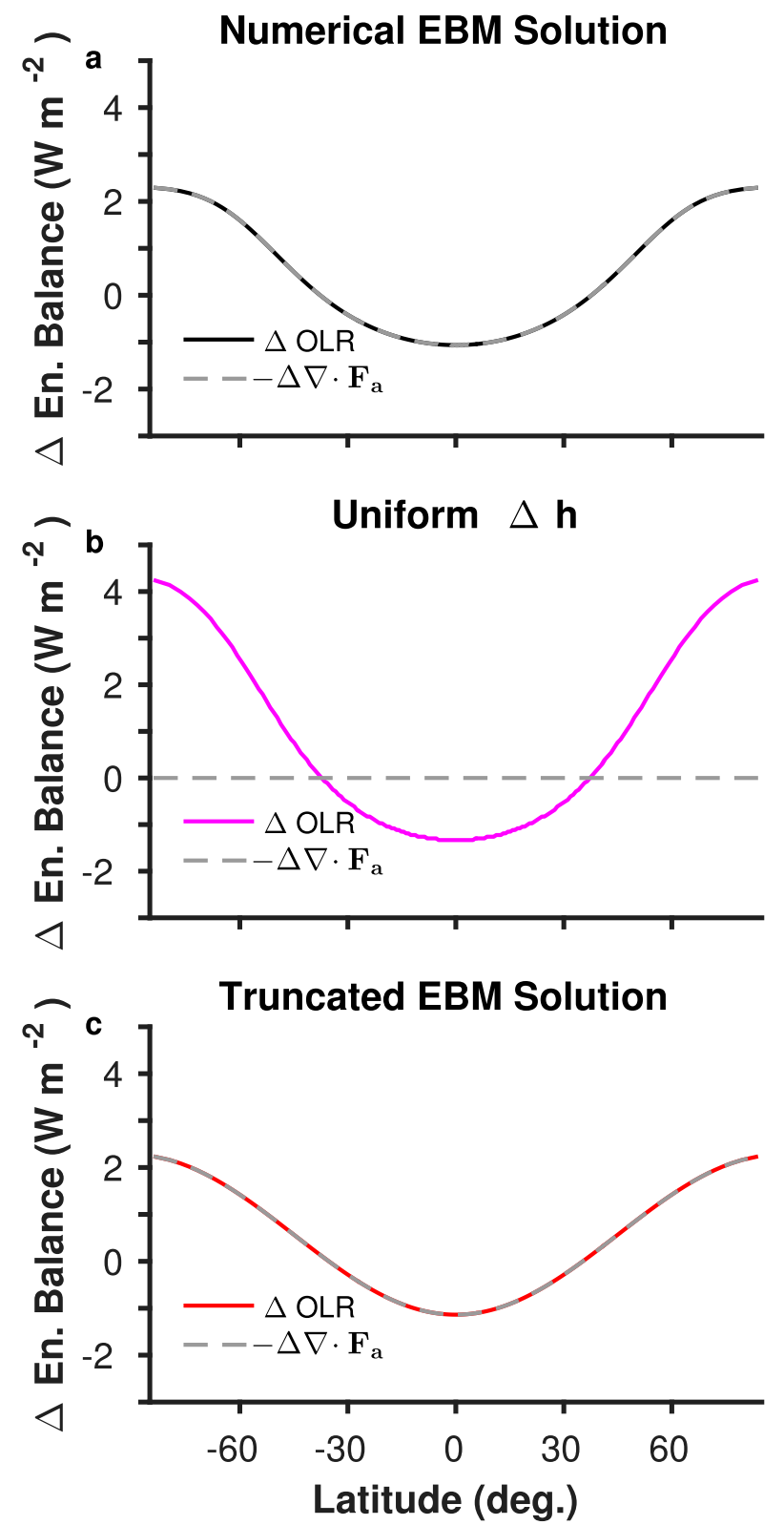

FIG. 3. Change in energy balance components for (a) the numerical EBM solutions, (b) the estimate assuming uniform increase in MSE from (2), and (c) the truncated solution to the EBM equation in (12).

\section{Simple estimates for polar amplification}

For all simple estimates, we neglect relative humidity changes, consistent with the numerical EBM solutions.

\section{a. Spatially uniform increase in MSE}

\section{1) Estimate}

Given the competition between the uniform warming's (that of a purely radiative warming) strengthened MSE gradients and the downgradient MSE diffusion, we consider the limit of a spatially uniform perturbation MSE. This is similar to the Byrne and O'Gorman (2013a) theory for land-sea contrast in surface warming, although spatial inhomogeneity in both the climatological and perturbation relative humidity played a central role there and are neglected here. Assuming the perturbation MSE is constant across latitude and separating the temperature into the climatology $\bar{T}(\phi)$ and departure thereof $\Delta T(\phi)$,

$\Delta h(\phi) \approx$ constant $=\Delta T(\phi)+L \mathcal{H} c_{p}^{-1} q^{*}[\bar{T}(\phi)+\Delta T(\phi)]$,

we can rearrange this expression symbolically to estimate the change in surface temperature:

$$
\Delta T(\phi) \approx \Delta\langle h\rangle-L \mathcal{H} c_{p}^{-1} q^{*}[\bar{T}(\phi)+\Delta T(\phi)],
$$

where the angle brackets denote the global mean. This is an implicit relationship because of the temperature dependence of the saturation specific humidity $q^{*}(T)$. In practice, we simply evaluate the change in MSE $\Delta h$ over a range of perturbation temperatures and find $\Delta T(\phi)$ that produces a spatially constant $\Delta h=\Delta\langle h\rangle$. We take $\Delta\langle h\rangle=5.2 \mathrm{~K}$ from the numerical EBM solutions, although approximating the increase in global-mean MSE using a uniform warming gives a comparable change $(5.6 \mathrm{~K})$.

The uniform perturbation MSE results in polaramplified warming because the high-latitude temperatures are sufficiently low that the latent energy makes a negligible contribution $\Delta T\left(\phi=90^{\circ}\right) \approx \Delta h$. In contrast, latent energy is a significant contribution to the energy content of low-latitude air. For $T \approx 300 \mathrm{~K}$ and $\mathcal{H}=80 \%$ as in Earth's tropics, $L \mathcal{H} \Delta q^{*} \approx 3 c_{p} \Delta T$. This implies that the low-latitude warming only needs to be one-quarter of the high-latitude warming to reach the same increase in MSE. In short, this estimate results in PA as a thermodynamic manifestation of cold trapping of the increase in energy: there is greater warming in high latitudes because nearly all of the increase takes the form of the temperature component of the energy content, which is a consequence of the low $q^{*}$ from the $\mathrm{CC}$ relation. This discussion can be formalized by considering a linearized definition of MSE [e.g., Rose et al. (2014) or the approach in section 3b] to obtain a uniform MSE PA estimate that depends on $\partial_{T} q^{*}$ evaluated at the equator and pole.

\section{2) Results}

Figure $1 \mathrm{~b}$ shows that this estimate has PA that is too large ( $\sim 3.4$ times more warming at pole than equator) compared to the numerical EBM solution $(\sim 2.3$ times more warming at pole than equator). This estimate's change in MSE is uniform by construction (Fig. 2b, 
magenta). The underlying assumption of the estimate that the change in MSE transport homogenizes the MSE is not realized in the numerical EBM solution (Fig. 2a, black). This is unsurprising given that the control simulation does not have a uniform MSE: diffusion does not succeed in homogenizing it for either the control or perturbation simulation. We note that this estimated warming pattern is not equivalent to numerical EBM solutions in the limit of large diffusivity. In that case, both the control and perturbation simulation are close to isothermal, so the warming is not strongly polar amplified.

The warming pattern of this estimate implies a polaramplified OLR change (magenta line in Fig. 3b), but the MSE flux should be unchanged according to the downgradient diffusion if the perturbation MSE is uniform $\left(\partial_{x} \Delta h=0\right)$. Therefore, this estimate does not satisfy the local TOA energy budget.

\section{b. Truncated solution to the moist EBM}

\section{1) Estimate}

Before proceeding to the truncated solution of the moist EBM, we review the component of North's (1975) spectral truncation to the constant-coefficient, dry EBM that is relevant to PA. The second Legendre polynomial $P_{2}$ coefficient of surface temperature $T_{2}$ is given by

$$
T_{2}=\frac{\left.\frac{1}{4} Q(S a)\right|_{2}}{6 \mathcal{D}+B},
$$

where the numerator is the second Legendre polynomial component of the absorbed solar radiation (indicated by the subscript 2), which can have contributions from the spatial structure of insolation $S(x)$ and planetary albedo $a(x)$. For the parameter values that we use, the second Legendre polynomial coefficient of the absorbed solar radiation is $-179.4 \mathrm{~W} \mathrm{~m}^{-2}$, and this would have an approximately $75-\mathrm{K}$ equator-pole temperature difference in a dry EBM $\left(T_{2}=-49.8 \mathrm{~K}\right.$ for the values of $B$ and $\mathcal{D}$ used here, although typically dry EBMs have larger values of $\mathcal{D}$, as described in what follows). Mathematically, the solution comes from the second-order polynomial component of the EBM governing equation and the eigenvalue of the diffusion operator giving the coefficient $6 \mathcal{D}$. Physically, we can consider the limit of $\mathcal{D} \rightarrow 0$, where the radiative equilibrium solution holds at each latitude and the steep equator-pole temperature contrast implies a large-amplitude, negative value of $T_{2}$. As $\mathcal{D}$ increases, the temperature becomes more spatially uniform: $T_{2} \rightarrow$ 0 as $\mathcal{D} \rightarrow \infty$. This is a formalization of the intuitive statement that the radiative equilibrium temperature has large meridional temperature gradients and the net effect of energy transport is to reduce the climatological equatorpole temperature contrast. With temperature-independent albedo, $T_{2}$ is climate invariant, but with Earth-like formulations for temperature-dependent albedo, the numerator in (3) becomes less negative under warming and gives rise to PA ( $T_{2}$ becomes less negative). For moist EBMs, there is PA with temperature-independent albedo and we generalize this solution to account for the latent energy transport.

To formulate the equivalent of (3) for the moist EBM, we must cope with the challenge of having different thermodynamic variables used for the diffusion and radiation, which give rise to the two terms in the denominator. We proceed to our new estimate by recognizing the MSE is a function of $T$ and linearize the definition of MSE about a spatially varying climatological temperature $\bar{T}(x)$ :

$$
\tilde{h} \equiv T+\frac{L \mathcal{H}}{c_{p}}\left[q^{*}(\bar{T})+\left.\frac{\partial q^{*}}{\partial T}\right|_{\bar{T}}(T-\bar{T})\right] .
$$

This is similar to the linearization performed in Rose et al. (2014), although we retain the climatological humidity $q^{*}(\bar{T})$ that they neglected because they only considered perturbed climate states. This approximate MSE (4) is used in the EBM's governing equation:

$$
C \partial_{t} T(\phi)=\frac{1}{4} Q S a-(A+B T)-\partial_{x}\left[\mathcal{D}\left(1-x^{2}\right) \partial_{x} \tilde{h}\right]+\mathcal{F} .
$$

Numerical EBM solutions with the linearized form of MSE $\tilde{h}$ agree well-in terms of PA-with the nonlinear form of MSE when the control EBM solution $\bar{T}(x)$ is used for both control and perturbation solution (Table 1).

Next, we expand the diffusion operator in terms of the components of MSE. After some cancellation, we have

$$
\begin{aligned}
\partial_{x}\left[\mathcal{D}\left(1-x^{2}\right) \partial_{x} \tilde{h}\right]= & \partial_{x}\left[\mathcal{D}\left(1+\left.\frac{L \mathcal{H}}{c_{p}} \frac{\partial q^{*}}{\partial T}\right|_{\bar{T}}\right)\left(1-x^{2}\right) \partial_{x} T\right] \\
& +\partial_{x}\left[\mathcal{D}\left(1-x^{2}\right)(T-\bar{T}) \partial_{x}\left(\left.\frac{L \mathcal{H}}{c_{p}} \frac{\partial q^{*}}{\partial T}\right|_{\bar{T}}\right)\right] .
\end{aligned}
$$

We consider how the two components of the MSE defined by (6) and (7) modify the dry EBM solution for the second Legendre polynomial component (3).

The first term (6) is the combination of the divergence of the dry energy flux and the climatological latent energy flux. The second term (7) represents changes in the latent energy flux divergence arising from departures 
from the climatological temperature. The form of the first term is equivalent to replacing the constant diffusivity in a temperature-diffusing EBM with a spatially varying diffusivity: $\mathcal{D}$ becomes $\mathcal{D}(x)=\mathcal{D}_{0} \eta(x)$ and $\eta(x)$ is defined as

$$
\eta(x) \equiv\left(1+\left.\frac{L \mathcal{H}}{c_{p}} \frac{\partial q^{*}}{\partial T}\right|_{\bar{T}}\right)
$$

Here, $\mathcal{D}_{0}$ has the same value $\left(0.3 \mathrm{~W} \mathrm{~m}^{-2} \mathrm{~K}^{-1}\right)$ as that of the spatially constant numerical EBM.

North (1975) presented the $P_{2}$ solution for the inhomogeneous diffusivity $\left[\mathcal{D}(x)=\mathcal{D}_{0} \eta(x)\right]$, which he envisioned as a representation of the Hadley circulation and aspects of atmospheric flows that arise from zonal asymmetries in boundary conditions. In other words, the components of the flow that are not transient eddies are poorly represented by constant coefficient downgradient diffusion. He showed that the solution ${ }^{1}$ took the form of (3) with $\mathcal{D}$ replaced by $\mathcal{D}^{\prime}$ :

$$
\mathcal{D}^{\prime}=-\frac{5}{6} \mathcal{D}_{0} \int_{0}^{1} P_{2}(x) \partial_{x}\left[\eta(x)\left(1-x^{2}\right) \partial_{x} P_{2}(x)\right] d x
$$

This is an integral that can be (numerically) computed once and used for both the control and perturbation climates as $\eta(x)$ depends on the climatological temperature $\bar{T}(x)$. Physically, the spatial inhomogeneity in $q^{*}$ leads to a larger diffusion coefficient $\mathcal{D}_{0} \eta$ in low latitudes and this results in increasing the effective diffusivity $\mathcal{D}^{\prime}$, which reduces the equator-pole temperature contrast $T_{2}$. For Earth-like $\bar{T}(x), \mathcal{D}^{\prime} \approx 2.4 \mathcal{D}_{0}$. One can think of this as the quantitative explanation for why previous authors have used empirically determined diffusivities in moist EBMs that are approximately a factor of 2 smaller than the standard dry values [as previously discussed in Flannery (1984)].

The second term that results from the expansion of MSE diffusion (7) depends on the anomalous temperature field for which we are solving. However, replacing the spatially varying $T-\bar{T}$ with its global-mean value $\Delta\langle T\rangle$ simplifies this term

$$
\begin{aligned}
\partial_{x}\left[\mathcal{D}\left(1-x^{2}\right)(T-\bar{T}) \partial_{x}\left(\left.\frac{L \mathcal{H}}{c_{p}} \frac{\partial q^{*}}{\partial T}\right|_{\bar{T}}\right)\right] \\
\quad \approx \partial_{x}\left[\mathcal{D}\left(1-x^{2}\right) \Delta\langle T\rangle \partial_{x}\left(\left.\frac{L \mathcal{H}}{c_{p}} \frac{\partial q^{*}}{\partial T}\right|_{\bar{T}}\right)\right],
\end{aligned}
$$

\footnotetext{
${ }^{1}$ North (1975) did not include the normalization factor of $6^{-1}$ in his equivalent of (8) and wrote the denominator of the inhomogeneous equivalent of (3) as $\mathcal{D}^{\prime}+B$.
}

and, as in previous estimates, we take $\Delta\langle T\rangle=\mathcal{F} / B$. This approximation assumes the spatial structure in the warming pattern is subdominant to the spatial gradient in the derivative of saturation specific humidity with respect to the climatological temperature. ${ }^{2}$ This is indeed true for the EBM simulations, and the subdominant role of spatial gradients in temperature anomalies in determining changes in the vertically integrated water vapor budget has previously been discussed (Boos 2012; Byrne and O'Gorman 2015). One can also use this approximation for the change in the latent energy flux convergence as the starting point for an additional simple estimate of polar amplification, which is described in appendix A.

The moist EBM equation (untruncated, with the linearized form of the $\mathrm{CC}$ relation) can be written as

$$
\begin{aligned}
& -\partial_{x}\left[\mathcal{D} \eta(x)\left(1-x^{2}\right) \partial_{x} T\right]+B T \\
& =\frac{1}{4} Q S a+\partial_{x}\left[\mathcal{D}\left(1-x^{2}\right) \Delta\langle T\rangle \partial_{x}\left(\partial_{T} q^{*} \mid \bar{T}\right)\right]-A+\mathcal{F} \\
& \quad \equiv \frac{1}{4} Q S a+\Delta \operatorname{LE}(x)-A+\mathcal{F}
\end{aligned}
$$

where we have arranged the $T$-dependent terms on the left-hand side of the equation and have placed the forcing terms on the right-hand side, which include the convergence of the latent energy flux resulting from the anomalous temperature $\Delta \operatorname{LE}(x)$.

At zeroth order (the global mean), the EBM solution is unaffected by the inhomogeneous diffusivity or the inclusion of latent energy:

$$
B T_{0}=\frac{1}{4} Q a_{0}-A+\mathcal{F} .
$$

There is no $\Delta \mathrm{LE}_{0}$ component because it is a flux divergence. The global-mean temperature is determined by the radiative balance between the global-mean absorbed shortwave [1/4 $\left(Q a_{0}\right)$, as $\left.S_{0}=1\right]$ and outgoing longwave $(A+B T-\mathcal{F})$ radiation.

At second order (indicated by the subscript 2 or $\left.\right|_{2}$ ), the left-hand side has the diffusivity multiplied by $\eta(x)$ and the right-hand side has $\Delta \mathrm{LE}_{2}$ :

$$
-\left.\partial_{x}\left[\mathcal{D} \eta(x)\left(1-x^{2}\right) \partial_{x} T\right]\right|_{2}+B T_{2}=\left.\frac{1}{4} Q(S a)\right|_{2}+\Delta \mathrm{LE}_{2} .
$$

The anomalous latent energy flux convergence term (7), computed using the approximation (9), combines with

\footnotetext{
${ }^{2}$ One can refine this statement by considering the spectral components of the terms in (7), as it is the $P_{2}$ component of the anomalous latent energy flux convergence that subsequently enters the PA estimate (12).
} 
the second Legendre polynomial component of the absorbed solar radiation in the numerator of the moist version of (3). Following the inhomogeneous diffusivity solution procedure (8), the second Legendre polynomial component of surface temperature for the moist EBM is

$$
T_{2}=\frac{\left.\frac{1}{4} Q(S a)\right|_{2}+\Delta \mathrm{LE}_{2}}{6 \mathcal{D}^{\prime}+B} .
$$

The temperature dependence of $T_{2}$, which leads to PA, arises from the temperature dependence of the humidity that enters in the numerator via the anomalous latent energy flux convergence $\Delta \mathrm{LE}_{2}$. The increase of $\Delta \mathrm{LE}_{2}$ with warming offsets part of the equator-pole contrast in absorbed solar radiation, some of the additional energy is locally balanced by radiation (about a third with standard values), and the remainder reduces the part of energy transport that is dependent on temperature gradients (6). Therefore, the equator-pole surface temperature contrast must decrease with warming.

This solution is solely a function of the EBM parameters $(\mathcal{D}, B$, and $\mathcal{F})$ and the climatological $\bar{T}(x)$. With $\bar{T}(x)$, a single numerical integral determines $\mathcal{D}^{\prime}$ and a spectral decomposition of the climatological humidity is used to determine $\Delta \mathrm{LE}_{2}$. This solution reproduces the $T_{2}$ of the control simulation $\left(\Delta \mathrm{LE}_{2}=0\right.$ if $\left.\mathcal{F}=0\right)$ and it captures the change in $T_{2}$ with warming. One can readily incorporate a spatially varying radiative forcing $\mathcal{F}(\phi)$, which simply affects the numerator of (12) (section 4). Prescribed spatially varying feedback parameters can also be incorporated by considering their second-order Legendre polynomial truncation in (11).

An alternative formulation of the moist EBM that leads to PA is to forgo the linearization of $\mathrm{CC}$ and write the $h=\left[1+L \mathcal{H} q^{*}(T)\left(c_{p} T\right)^{-1}\right] T$ (Flannery 1984), which leads to a temperature-dependent $\mathcal{D}^{\prime}(T)$ because $\eta(x)$ changes with climate. We can write the truncated solution as

$$
T_{2}=\frac{\left.\frac{1}{4} Q(S a)\right|_{2}}{6 \mathcal{D}^{\prime}(T)+B},
$$

where the numerical integral $\mathcal{D}^{\prime}(8)$ must be recomputed for the perturbed climate, but there is no $\Delta \mathrm{LE}_{2}$ in the numerator. From this perspective, discussed by Flannery (1984), PA results from the changes in latent energy giving rise to a larger effective diffusivity $\mathcal{D}^{\prime}$ with warming, which reduces the magnitude of $T_{2}$.

\section{2) Results}

Using the EBM control $\bar{T}(x), \Delta \mathrm{LE}_{2}=0, \mathcal{D}^{\prime}=$ $4.2 \mathrm{~W} \mathrm{~m}^{-2} \mathrm{~K}^{-1}$, and $T_{2}=-29.7 \mathrm{~K}$, implying an equatorpole contrast $\Delta_{h} T \approx 45 \mathrm{~K}$. This is close to the numerical EBM (Table 1). The truncated solution has a temperature change pattern that is nearly identical to the numerical EBM solutions (Fig. 1c). The anomalous convergence of latent energy is $\Delta \mathrm{LE}_{2}=7.6 \mathrm{~W} \mathrm{~m}^{-2}$, offsetting approximately $4 \%$ of the second Legendre polynomial component of the absorbed solar radiation. There are minor deviations that can arise from the nonzero higher-order Legendre polynomials in the numerical EBM solution (Table 1), from linearizing CC, and from the approximation of neglecting the spatial structure of the temperature change to compute $\Delta \mathrm{LE}_{2}$ [(9)]. Likewise, the MSE perturbation is close to the numerical EBM solution (red vs black in Fig. 2) and the changes in both radiative fluxes and MSE flux divergence of the estimate are almost identical to the numerical EBM solution (Figs. 3a,c).

The evaluation of the truncated EBM solution (12) for small climate changes requires a numerical evaluation of the anomalous latent energy flux convergence (9), which is a function of the climatological temperature distribution. Ideally, one could approximately estimate polar amplification without evaluating spatial gradients of the saturation specific humidity and spectrally truncating the results. With that goal in mind, we derive a further approximation to $\Delta \mathrm{LE}_{2}$ in appendix $\mathrm{B}$. The resulting expression,

$$
\Delta \mathrm{LE}_{2} \approx-\left.6 T_{2} \mathcal{D} \Delta\langle T\rangle L \mathcal{H} c_{p}^{-1} \partial_{T T} q *\right|_{\langle T\rangle},
$$

depends on the EBM coefficients $(\mathcal{D}, \mathcal{F}, B$, and $\mathcal{H})$, thermodynamic constants ( $L$ and $c_{p}$ ), the climatological $T_{2}$, and the second derivative of the saturation specific humidity with respect to temperature evaluated at the global-mean temperature $\left.\partial_{T T} q *\right|_{\langle T\rangle}$. Substituting this approximation into (12) leads to a relationship for the sensitivity of the change in $T_{2}$ per unit of global temperature change $\left(\Delta\langle T\rangle \equiv \Delta T_{0}\right)$ :

$$
\frac{\Delta T_{2}}{\Delta\langle T\rangle}=\frac{-\left.6 T_{2} \mathcal{D} L \mathcal{H} c_{p}^{-1} \partial_{T T} q^{*}\right|_{\langle T\rangle}}{6 \mathcal{D}^{\prime}+B} .
$$

Evaluating this approximation for the control EBM temperature $\left(T_{2}=-29.3 \mathrm{~K}\right.$ and $\left.\left.\partial_{T T} q *\right|_{\langle T\rangle} \approx 4.3 \times 10^{-5} \mathrm{~K}^{-2}\right)$ implies a polar warming of 2.8 times that of the equator, somewhat larger than the numerical EBM's factor of 2.3. This further approximation of $\Delta \mathrm{LE}_{2}$ helps anticipate the results of the next section, where we examine the truncated solution of the PA estimate for a wide range of climatological temperatures.

\section{Applications of the truncated solution}

\section{a. Wide range of climates}

We perform a series of numerical EBM calculations with a wide range $\left(50 \mathrm{~W} \mathrm{~m}^{-2}\right)$ of uniform radiative forcing to examine how PA varies from substantially 

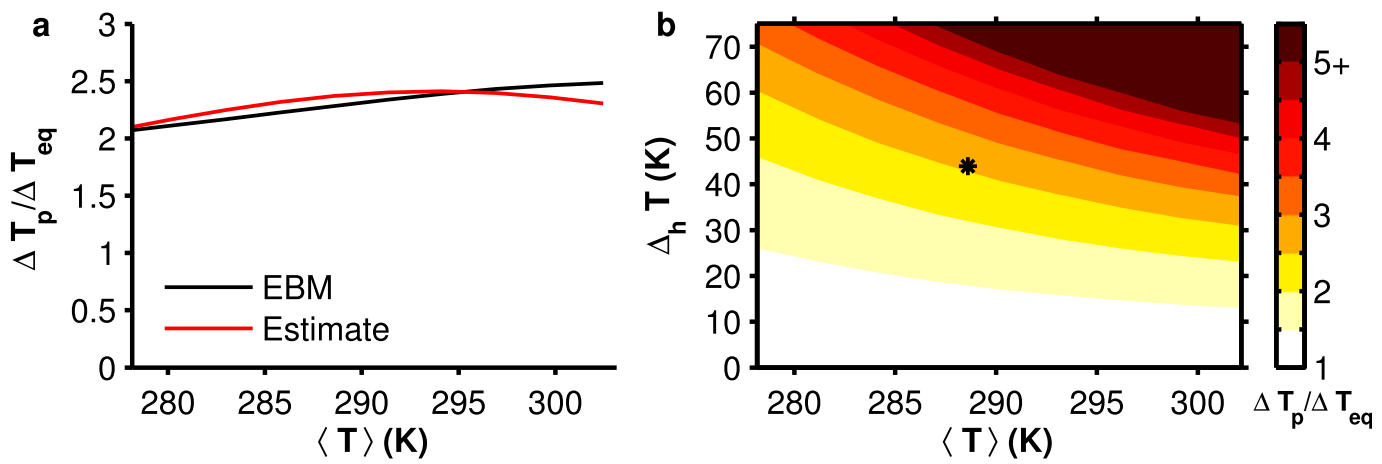

FIG. 4. Polar amplification for (a) the numerical EBM solutions and truncated solution over a wide range of spatially uniform radiative forcing and (b) the truncated EBM solution over a wide range of synthetic climatological temperatures. The asterisk in (b) shows where the control numerical EBM solution is in this parameter space.

colder to substantially warmer climates. There is a weak increase in PA over the wide range of forcing (Fig. 4a). As the climate warms, the moist EBM has increased global-mean temperature, which tends to increase the latent energy flux convergence, and decreased equatorpole temperature contrast, which tends to decrease the latent energy flux convergence. These competing changes result in polar amplification's weak dependence over the range of forcing values.

We use temperatures of the numerical EBM solutions to compute the spectral estimate for each value of radiative forcing. The truncated estimate for PA is broadly similar to the numerical EBM solutions (Fig. 4a). It is slightly larger than the PA of numerical EBM solution in colder climates and slightly smaller in warmer climates, even though in all cases the estimate is within $10 \%$ of the numerical EBM solution. For large climate changes, changes in both the latent energy flux convergence $\Delta \mathrm{LE}_{2}$ and the effective diffusivity $\mathcal{D}^{\prime}$ are important. Here, they vary as the mean state changes at a similar rate ( $\sim 3 \% \mathrm{~K}^{-1}$ in the Earth-like range of climates). The weak positive dependence of PA on the global-mean temperature then arises because the changes in the denominator of (12), the combination of the effective diffusivity and the invariant feedback parameter $B$, do not keep pace with changes in the numerator of (12).

The truncated estimate can readily be evaluated over a wide range of climatological temperature distributions to examine the controls on the magnitude of polar amplification. Here, we use an idealized second Legendre polynomial formula for $\bar{T}(x)=T_{0}+T_{2} P_{2}(x)$ and systematically vary the global-mean temperature $T_{0}$ and the equator-pole temperature contrast $\Delta_{h} T=1.5 T_{2}$ to generate synthetic climatological temperatures. These climatological temperatures, which could be interpreted as arising from insolation changes, are used to estimate polar amplification using (12) and the other parameter values as before. Figure $4 \mathrm{~b}$ shows that PA increases as the global-mean temperature increases and as the equatorpole temperature contrast increases, as can be deduced from (14). For unchanged $T_{2}$, increased $T_{0}$ gives rise to greater PA because $\Delta \mathrm{LE}_{2}$ is larger as a result of CC: the same temperature contrast implies a greater humidity contrast at warmer temperatures. For unchanged $T_{0}$, increased $\Delta_{h} T$ gives rise to greater PA because $\Delta \mathrm{LE}_{2}$ grows larger with the equator-pole temperature contrast and the concomitant humidity contrast. The estimate's increasing PA with equator-pole contrast provides a qualitative guide to the seasonal cycle of polar amplification arising from changes in latent energy transport: winter $\left(\Delta_{h} T \approx 50 \mathrm{~K}\right)$ would be expected to have about $60 \%$ more PA than summer $\left(\Delta_{h} T \approx 30 \mathrm{~K}\right)$. We note that a full account of the seasonal cycle of PA requires consideration of more than the role that the seasonally varying temperature gradient plays in determining the changes in latent energy transport (Kim et al. 2018).

\section{b. Solar versus greenhouse gas forcing}

The possibility that the climate system responds differently to solar and greenhouse gas $(\mathrm{GHG})$ radiative forcing has been investigated in GCM simulations (e.g., Govindasamy and Caldeira 2000; Boer and Yu 2003; Hansen et al. 2005; Merlis et al. 2014). Differences across forcing agents would have important implications for solar radiation management (SRM) geoengineering schemes, where the warming from the positive radiative forcing of increased GHG concentration is offset by a negative radiative forcing from, for example, sulfate aerosols in the stratosphere. In idealized geoengineering simulations where the negative radiative forcing is imposed simply by reducing the solar constant, there is residual polar warming (Govindasamy and Caldeira 2000; Kravitz et al. 2013; Russotto and Ackerman 2018). 
In other words, the temperature change pattern from GHGs is more polar amplified than that of solar forcing. The residual polar warming has been argued to result from differences in radiative forcing (Govindasamy and Caldeira 2000; Kravitz et al. 2013; Russotto and Ackerman 2018), as has also been shown to be important for intertropical convergence zone shifts (Viale and Merlis 2017; Russotto and Ackerman 2018), rather than forcing agent dependence in radiative feedbacks. This suggests that the moist EBM with a specified feedback parameter is a viable minimal framework to address the residual polar warming.

Here, we make use of the new truncated EBM solution to straight forwardly estimate the difference in the spatial pattern of forcing on temperature change. We now include the spatial structure of the radiative forcing in the second-order truncation, which leads to

$$
T_{2}=\frac{\frac{1}{4} Q(S a)_{2}+\Delta \mathrm{LE}_{2}+\mathcal{F}_{2}}{6 \mathcal{D}^{\prime}+B} .
$$

The truncated solution is assessed for the combination of positive GHG forcing and negative solar forcing that has zero global-mean radiative forcing. The radiative forcing of a solar constant change depends on the insolation and planetary albedo $\mathcal{F}^{S}=1 / 4[\Delta Q S(x) a(x)]$, with the superscript indicating the forcing agent. The zeroth- and second-order components of the solar radiative forcing are $\mathcal{F}^{S}=\mathcal{F}_{0}^{S}+\mathcal{F}_{2}^{S}=1 / 4\left(\Delta Q a_{0}\right)+$ $1 / 4\left[\Delta Q\left(a_{0} \gamma+a_{2}\right)\right] P_{2}$. The idealization of spatially uniform longwave forcing used in the previous sections is not a quantitatively accurate representation of carbon dioxide's radiative forcing of Earth's climate (e.g., Huang et al. 2016). The second-order spatial structure of carbon dioxide is $\mathcal{F}_{2}^{G}=\chi \mathcal{F}_{0}^{G}$ with $\chi \approx-0.5$; that is, the forcing at the equator is about 2.5 times that of the pole independent of the magnitude of the globalmean radiative forcing. For the SRM scheme, the global-mean solar radiative forcing is equal in magnitude and opposite in sign of the carbon dioxide forcing: $\mathcal{F}_{0}^{S}=-\mathcal{F}_{0}^{G}$. This implies that, at second order, $\mathcal{F}_{2}^{G}-\mathcal{F}_{2}^{S}=$ $\mathcal{F}_{0}^{G}\left[\chi-\left(a_{2} / a_{0}+\gamma\right)\right]$. This expression is a difference between the second-order structure of the forcing between the positive carbon dioxide forcing and negative solar forcing, which enters the numerator of the expression for the second-order component of temperature (15). If the quantity in square brackets is positive, this implies a larger magnitude forcing contrast for solar than carbon dioxide forcing, which will provoke residual polar warming $\left(T_{2}\right.$ increases). For Earth's climate, $a_{2} / a_{0}+$ $\gamma \approx-0.8$ and is indeed larger magnitude than $\chi \approx-0.5$. Given the approximation of the perturbation latent energy flux convergence (9) is adequate, the geoengineered climate state has $\Delta \mathrm{LE}_{2}=0$ because $\mathcal{F}_{0}^{S}=-\mathcal{F}_{0}^{G}$ and the moist EBM estimate reduces to a dry EBM estimate. Therefore, a quadrupling of carbon dioxide with $\mathcal{F}_{0}^{G} \approx$ $7 \mathrm{~W} \mathrm{~m}^{-2}$ implies $\Delta_{\mathrm{SRM}} T_{2} \approx 0.3 \mathrm{~K}$, using $6 \mathcal{D}^{\prime}+B=$ $6.2 \mathrm{~W} \mathrm{~m}^{-2} \mathrm{~K}^{-1}$ as before. Numerical EBM solutions subjected to SRM forcing with $\chi \approx-0.5, \mathcal{F}_{0}^{G}=7.0 \mathrm{~W} \mathrm{~m}^{-2}$, and solar radiation parameters $\left(\gamma, a_{0}\right.$, and $\left.a_{2}\right)$ as before result in $T_{2}=0.35 \mathrm{~K}$, in good agreement with the analytic estimate.

The ensemble mean of GCMs participating in the Geoengineering Model Intercomparison Project (GeoMIP) G1 experiment, which has a quadrupling of carbon dioxide and corresponding decrease in solar constant, has $\Delta_{\mathrm{SRM}} T_{2} \approx 0.7 \mathrm{~K}$ (Russotto and Ackerman 2018). The higher value of residual polar amplification in the GeoMIP G1 GCM simulations likely results from a robust spatially varying feedback parameter that varies between equator and pole (e.g., surface albedo and lapse rate feedbacks that are zero or stabilizing in low latitudes and are destabilizing in high latitudes) and amplifies the response we obtained analytically with a uniform feedback parameter. Nevertheless, the analytic estimate qualitatively captures the GCM behavior.

\section{Conclusions}

Moist energy balance models (EBMs) provide an idealized framework to investigate polar amplification (PA). Even with spatially uniform radiative forcing and feedbacks, PA can arise as a consequence of increased energy transport with warming that results from increasing humidity gradients-a consequence of the Clausius-Clapeyron (CC) relation. Moist EBM simulations typically have about a factor of 2 more warming at the pole than the equator (Flannery 1984), although this can be modulated by spatial structure in radiative feedbacks (Roe et al. 2015). To understand the magnitude of PA in moist EBMs, we present two simple estimates and compare their pattern of warming, pattern of change in moist static energy, and TOA budget change to those of numerical moist EBM solutions.

The first estimate (section 3a) is based on converting a uniform change in MSE into a surface temperature change. This is what the anomalous MSE would be if the perturbation energy flux divergence was perfectly efficient at removing the perturbation MSE gradient. Note that Earth-like values of the diffusivity have nonzero gradients in the control climate's MSE, so one would not expect the perturbation MSE gradient to be zero. This estimate has a factor of 3.4 more warming at the pole than the equator, a substantial overestimate compared to the numerical EBM solution. This estimate also has an inconsistent TOA budget change: there is an increase 
in polar OLR from the locally enhanced warming, which would need to be balanced by an increased energy flux convergence. However, a uniform MSE perturbation would not alter the energy flux convergence, according to the EBM's downgradient relationship.

The second estimate (section $3 \mathrm{~b}$ ) is based on truncating the moist EBM at the second Legendre polynomial $\left(P_{2} \propto \sin ^{2} \phi\right)$ component of the solution, which dominates the warming pattern and gives rise to PA. For this, we can adapt the spectral solution techniques of North (1975) that were developed for dry EBMs (where temperature, rather than MSE, is diffused), and cast the modification that arises from including latent energy as a spatially varying diffusivity and, for perturbed climates, a latent energy flux convergence component that offsets the planetary-scale absorbed solar radiation gradient. The increased high-latitude energy convergence is not completely locally balanced by warming sufficient to radiatively rebalance the TOA budget. Rather, there is some of the anomalous latent energy flux convergence (approximately one-third) that is compensated for by a decrease in the temperature-dependent energy transport. This truncated solution captures the numerical EBM simulation results and depends only on the climatological temperature, which is needed to determine the change in latent energy transport, and EBM parameters like the longwave feedback parameter $B$ and diffusivity $\mathcal{D}$.

What is the physical insight from the estimate that does not agree quantitatively with the numerical EBM solutions? One key result from the uniform MSE perturbation estimate (section 3a) is that the moist EBM's equilibrium climate states are not in the regime where the downgradient diffusion succeeds at diffusing the anomalous MSE gradient away; rather, these climate states are the result of the competition between the atmosphere's radiative fluxes and the downgradient diffusion that leads to MSE anomalies that are between the limiting cases of the uniform temperature change's MSE anomalies and uniform MSE perturbations (Fig. 2). The truncated solution accounts for the partially compensating decrease of the temperature-dependent (dry) energy transport in response to the increase in the latent energy flux, which is estimated by assuming a uniform warming. In short, the successful truncated solution shows that the magnitude of the PA depends on the relative strength of the radiative restoring and the temperature dependence of both dry and moist components of the energy transport changes. We note that partial compensation between changes in dry and latent energy transports in the midlatitudes has been widely discussed in GCM simulations (e.g., Held and Soden 2006; O'Gorman and Schneider 2008), so it is noteworthy that this behavior is present in the much simpler
EBM simulations, where we see that the magnitude of the partial compensation is directly connected to the spatial pattern of warming.

The truncated solution can readily be applied to climate change questions and offers a GCM-independent expectation for these changes. We present an application to a wide range of climate states. This sheds light into the dependence of PA on the climatological temperature distribution. First, polar amplification is weak in climate states with weak temperature gradients. Second, climate states that are warmer have more polar amplification, all else equal. These sensitivities are captured in the approximate formula for the change in latent energy flux convergence (13) derived in appendix B. In the moist EBM, the globalmean and equator-pole temperature contrasts covary in a manner that implies limited changes in PA as radiative forcing is successively increased.

We have also evaluated a version of the truncated spectral solution that includes spatial structure in the radiative forcing to assess the magnitude of "residual" polar warming in solar radiation management geoengineering scenarios. Similar to numerical EBM solutions, the estimate suggests approximately $0.3 \mathrm{~K}$ of residual polar warming for quadrupled carbon dioxide forcing with an offsetting reduction in the solar constant. This is about half as large as the ensemble mean results of the similarly forced GeoMIP G1 GCM simulations (Russotto and Ackerman 2018), implying an amplifying role for spatially varying feedbacks in GCMs. These two applications of the truncated spectral solution are suggestive of its broader value in addressing climate change problems.

The simple estimates for PA can easily be evaluated in EBMs, where parameters such as the diffusivity are imposed. For Earth's climate, one could examine the truncated estimate's change in latent energy flux divergence (section $3 \mathrm{~b}$ ) by applying a simple ClausiusClapeyron-based multiplicative amplification factor (Held and Soden 2006) to reanalysis estimates of the latent energy flux divergence. In addition, parameters such as the longwave feedback parameter and diffusivity (12) that would also need to be empirically determined. The warming pattern that results in a uniformly increased MSE (section 3a) is readily applied to observational estimates, as it only depends on the climatology of the surface air temperature and relative humidity. This estimate may perform better for Earth's atmosphere than for the moist EBM because it approximately accounts for the destabilizing high-latitude lapse rate and surface albedo feedbacks that are absent from the EBM simulations presented here. That is, it can be viewed as more than an estimate for the energy transport when the vertical structure of the atmosphere can vary and respond to surface boundary condition changes. 
Here, we have presented two simple estimates for PA in the moist EBM with climate-invariant surface albedo. Given the importance of spatially varying feedbacks like the lapse rate and surface albedo feedbacks, an important next step is to revisit the truncated moist EBM solution with prescribed spatially varying radiative feedbacks.

Acknowledgments. We thank Paul O'Gorman for suggesting the uniform perturbation moist static energy estimate, Nadir Jeevanjee for his encouragement, and Tim Cronin for pointing out that spatially varying feedbacks can be incorporated in the truncated EBM solution. Tim Cronin and two anonymous reviewers provided helpful feedback on the presentation of the results. This work was supported by the Fonds de Recherche du Québec Nature et Technologies (FRQNT) Nouveau Chercheur Award 197873 and Natural Science and Engineering Research Council (NSERC) Grant RGPIN-2014-05416.

\section{APPENDIX A}

\section{Increased MSE Transport from Uniform Warming}

An additional estimate for the pattern of warming can be obtained by considering the increase in energy transport from a uniform warming, following Shaw and Voigt's (2016) examination of general circulation changes in uniform perturbation sea surface temperature GCM simulations. The uniform warming is the temperature change for a locally balanced perturbation radiation budget in the moist EBM, so one can envision the superposition of the uniform warming with the patterned response that arises from the increase in MSE flux divergence (cooling the tropics and warming the poles). Because the uniform warming does not change the temperature gradient $\left(\partial_{x} T^{\prime}=\partial_{x} \bar{T}\right)$, the perturbation energy flux divergence solely arises from the anomalous latent energy flux divergence. This perturbation energy flux convergence is then converted to a temperature change by determining the change sufficient to locally rebalance the TOA budget $\mathcal{F}-\Delta\left(\nabla \cdot \mathbf{F}_{a}\right) \approx B \Delta T$. The fidelity of the estimate for PA then depends on the closeness of the EBM simulated change in energy flux divergence with that of the increase given by this CC-based estimate.

This estimate excites higher-order spectral components of larger amplitude than in the numerical EBM solutions and also has a substantially larger degree of polar amplification (comparable to the uniform MSE change estimate; section 3a) than in the numerical EBM solutions. This estimate is similar to the truncated spectral solution in that both have an increase in latent energy flux convergence governed by CC. The critical difference is that the truncated solution accounts for the partially compensating decrease of the temperaturedependent (dry) energy transport in response to the increase in the latent energy flux. Mathematically, the larger magnitude PA for the estimate described in this appendix can be understood by considering it as the limit of vanishing diffusivity $\mathcal{D}^{\prime} \rightarrow 0$ of the perturbation spectral solution (12). This estimate, therefore, breaks the relationship between the mean MSE gradient and the energy flux: the assumed increase in the MSE flux $\left(\propto \mathcal{D} \partial_{x} h\right)$ is sufficient to decrease the mean MSE gradient $\partial_{x} h$ implied by the estimated temperature change.

\section{APPENDIX B}

\section{Further Approximation to the Change in Latent Energy Flux}

The approximate change in latent energy flux convergence (9) used in the truncated solution,

$$
\Delta \mathrm{LE}(x) \equiv \mathcal{D} \Delta\langle T\rangle L \mathcal{H} c_{p}^{-1} \partial_{x}\left[\left(1-x^{2}\right) \partial_{x}\left(\left.\frac{\partial q^{*}}{\partial T}\right|_{\bar{T}}\right)\right],
$$

can be written as

$$
\Delta \mathrm{LE}(x)=\mathcal{D} \Delta\langle T\rangle L \mathcal{H} c_{p}^{-1} \partial_{x}\left[\left(1-x^{2}\right) \partial_{x} \bar{T} \partial_{T T} q^{*}\right]
$$

using the chain rule.

We are interested in the second-order component of $\Delta \mathrm{LE}$, and we assume it can be approximated using the second-order truncation of temperature: $\bar{T}(x)=T_{0}+$ $T_{2} P_{2}(x)$. This is an error of $8 \%$ for the control EBM temperature. Computing the first $x$-derivative in $\Delta \mathrm{LE}(x)$ results in the following expression:

$$
\begin{aligned}
\Delta \mathrm{LE}(x)= & \mathcal{D} \Delta\langle T\rangle L \mathcal{H} c_{p}^{-1}\left[\left(1-x^{2}\right) \partial_{x x} \bar{T} \partial_{T T} q^{*}\right. \\
& \left.-2 x \partial_{x} \bar{T} \partial_{T T} q^{*}\right],
\end{aligned}
$$

into which we then substitute the above form of $\bar{T}(x)$. After collecting terms and replacing polynomials in $x$ with the appropriate Legendre polynomials, we obtain

$$
\Delta \mathrm{LE}(x)=-6 T_{2} \mathcal{D} \Delta\langle T\rangle L \mathcal{H} c_{p}^{-1} P_{2} \partial_{T T} q^{*} .
$$

Now, a spectral truncation at second order is performed to determine $\Delta \mathrm{LE}_{2}$. The truncation of the product of the second Legendre polynomial and the second derivative of the saturation specific humidity with respect to temperature is approximately given by $\left.\left.\left(P_{2} \partial_{T T} q *\right)\right|_{2} \approx \partial_{T T} q *\right|_{0}$. This results in

$$
\Delta \mathrm{LE}_{2} \approx-\left.6 T_{2} \mathcal{D} \Delta\langle T\rangle L \mathcal{H} c_{p}^{-1} \partial_{T T} q *\right|_{0} .
$$


This is about a $30 \%$ overestimate for Earth-like temperatures as there are neglected second-order components of $\left.P_{2} \partial_{T T} q *\right|_{2}$ and $\left.P_{2} \partial_{T T} q *\right|_{4}$ that reduce $\Delta \mathrm{LE}_{2}$. One further approximation is to evaluate $\partial_{T T} q^{*}$ at the globalmean temperature rather than evaluating it locally before taking the global mean. This results in the expression (13) written in section $3 \mathrm{~b}$ :

$$
\Delta \mathrm{LE}_{2} \approx-\left.6 T_{2} \mathcal{D} \Delta\langle T\rangle L \mathcal{H} c_{p}^{-1} \partial_{T T} q^{*}\right|_{\langle T\rangle} .
$$

This expression has explicit dependence on $T_{2}$, with larger magnitude equator-pole temperature contrast increasing $\Delta \mathrm{LE}_{2}$, and an implicit dependence on the global-mean temperature $\langle T\rangle=T_{0}$ through the saturation specific humidity. The global-mean temperature dependence is positive, as $\partial_{T T} q^{*}$ is an increasing function of temperature.

For the second-order truncation of the Earth-like control climate (Table 1), $\Delta \mathrm{LE}_{2}=8.2 \mathrm{~W} \mathrm{~m}^{-2}$. Evaluating the approximate formula (13) with $\left.\partial_{T T} q^{*}\right|_{\langle T\rangle} \approx 4.3 \times 10^{-5} \mathrm{~K}^{-2}$ results in $\Delta \mathrm{LE}_{2} \approx 9.0 \mathrm{~W} \mathrm{~m}^{-2}$. There are canceling errors from neglecting the projection of higher-order components of $\partial_{T T} q^{*} P_{2}$ and evaluating $\partial_{T T} q^{*}$ using the global-mean temperature rather than evaluating it locally and then taking its global mean. The resulting estimate of the change in $T_{2}$ of $0.7 \mathrm{~K} \mathrm{~K}^{-1}$ of global-mean temperature change is modestly larger than the numerical EBM solution (Table 1), so the pole is estimated to warm 2.8 times as much as the equator compared to the numerical EBM's factor of 2.3.

\section{REFERENCES}

Alexeev, V. A., 2003: Sensitivity to $\mathrm{CO}_{2}$ doubling of an atmospheric GCM coupled to an oceanic mixed layer: A linear analysis. Climate Dyn., 20, 775-787, https://doi.org/10.1007/ s00382-003-0312-x.

Boer, G., and B. Yu, 2003: Climate sensitivity and response. Climate Dyn., 20, 415-429, https://doi.org/10.1007/s00382-002-0283-3.

Boos, W. R., 2012: Thermodynamic scaling of the hydrological cycle of the Last Glacial Maximum. J. Climate, 25, 992-1006, https://doi.org/10.1175/JCLI-D-11-00010.1.

Byrne, M. P., and P. A. O'Gorman, 2013a: Land-ocean warming contrast over a wide range of climates: Convective quasiequilibrium theory and idealized simulations. J. Climate, 26, 4000-4016, https://doi.org/10.1175/JCLI-D-12-00262.1.

_ , and _ 2013b: Link between land-ocean warming contrast and surface relative humidities in simulations with coupled climate models. Geophys. Res. Lett., 40, 5223-5227, https:// doi.org/10.1002/grl.50971.

_ , and — 2015: The response of precipitation minus evapotranspiration to climate warming: Why the "wet-get-wetter, dry-get-drier" scaling does not hold over land. J. Climate, $\mathbf{2 8}$, 8078-8092, https://doi.org/10.1175/JCLI-D-15-0369.1.

Caballero, R., and J. Hanley, 2012: Midlatitude eddies, stormtrack diffusivity, and poleward moisture transport in warm climates. J. Atmos. Sci., 69, 3237-3250, https://doi.org/ 10.1175/JAS-D-12-035.1.
Collins, M., and Coauthors, 2013: Long-term climate change: Projections, commitments and irreversibility. Climate Change 2013: The Physical Science Basis, T. F. Stocker et al., Eds., Cambridge University Press, 1029-1136.

Feldl, N., and G. H. Roe, 2013: The nonlinear and nonlocal nature of climate feedbacks. J. Climate, 26, 8289-8304, https://doi.org/ 10.1175/JCLI-D-12-00631.1.

Flannery, B. P., 1984: Energy balance models incorporating transport of thermal and latent energy. J. Atmos. Sci., 41, 414-421, https:// doi.org/10.1175/1520-0469(1984)041<0414:EBMITO>2.0.CO;2.

Frierson, D. M. W., I. M. Held, and P. Zurita-Gotor, 2007: A grayradiation aquaplanet moist GCM. Part II: Energy transports in altered climates. J. Atmos. Sci., 64, 1680-1693, https://doi.org/ 10.1175/JAS3913.1.

Govindasamy, B., and K. Caldeira, 2000: Geoengineering Earth's radiation balance to mitigate $\mathrm{CO}_{2}$-induced climate change. Geophys. Res. Lett., 27, 2141-2144, https://doi.org/10.1029/ 1999GL006086.

Hall, A., 2004: The role of surface albedo feedback in climate. J. Climate, 17, 1550-1568, https://doi.org/10.1175/ 1520-0442(2004)017<1550:TROSAF $>2.0$. CO;2.

Hansen, J., and Coauthors, 2005: Efficacy of climate forcings. J. Geophys. Res., 110, D18104, https://doi.org/10.1029/2005JD005776.

—, R. Ruedy, M. Sato, and K. Lo, 2010: Global surface temperature change. Rev. Geophys., 48, RG4004, https://doi.org/ 10.1029/2010RG000345.

Held, I. M., and B. J. Soden, 2006: Robust responses of the hydrological cycle to global warming. J. Climate, 19, 5686-5699, https://doi.org/10.1175/JCLI3990.1; Corrigendum, 24, 15591560, https://doi.org/10.1175/2010JCLI4045.1.

Huang, Y., and M. Zhang, 2014: The implication of radiative forcing for meridional energy transport. Geophys. Res. Lett., 41, 1665-1672, https://doi.org/10.1002/2013GL059079.

, X. Tan, and Y. Xia, 2016: Inhomogeneous radiative forcing of homogeneous greenhouse gases. J. Geophys. Res. Atmos., 121, 2780-2789, https://doi.org/10.1002/2015JD024569.

Hwang, Y.-T., D. M. W. Frierson, and J. E. Kay, 2011: Coupling between Arctic feedbacks and changes in poleward energy transport. Geophys. Res. Lett., 38, L17704, https://doi.org/ 10.1029/2011GL048546.

Kang, S. M., D. M. W. Frierson, and I. M. Held, 2009: The tropical response to extratropical thermal forcing in an idealized GCM: The importance of radiative feedbacks and convective parameterization. J. Atmos. Sci., 66, 2812-2827, https:// doi.org/10.1175/2009JAS2924.1.

Kim, D., S. M. Kang, Y. Shin, and N. Feldl, 2018: Sensitivity of polar amplification to varying insolation conditions. J. Climate, 31, 4933-4947, https://doi.org/10.1175/JCLI-D-17-0627.1.

Kravitz, B., and Coauthors, 2013: Climate model response from the Geoengineering Model Intercomparison Project (GeoMIP). J. Geophys. Res. Atmos., 118, 8320-8332, https://doi.org/ 10.1002/jgrd.50646.

Lee, S., 2014: A theory for polar amplification from a general circulation perspective. Asia-Pac. J. Atmos. Sci., 50, 31-43, https://doi.org/10.1007/s13143-014-0024-7.

Merlis, T. M., 2014: Interacting components of the top-ofatmosphere energy balance affect changes in regional surface temperature. Geophys. Res. Lett., 41, 7291-7297, https:// doi.org/10.1002/2014GL061700.

, I. M. Held, G. L. Stenchikov, F. Zeng, and L. W. Horowitz, 2014: Constraining transient climate sensitivity using coupled climate model simulations of volcanic eruptions. J. Climate, 27, 7781-7795, https://doi.org/10.1175/JCLI-D-14-00214.1. 
North, G. R., 1975: Theory of energy-balance climate models. J. Atmos. Sci., 32, 2033-2043, https://doi.org/10.1175/ 1520-0469(1975)032<2033:TOEBCM>2.0.CO;2.

— , R. F. Cahalan, and J. A. Coakley Jr., 1981: Energy balance climate models. Rev. Geophys., 19, 91-121, https://doi.org/ 10.1029/RG019i001p00091.

O'Gorman, P. A., and T. Schneider, 2008: The hydrological cycle over a wide range of climates simulated with an idealized GCM. J. Climate, 21, 3815-3832, https://doi.org/10.1175/ 2007JCLI2065.1.

Pithan, F., and T. Mauritsen, 2014: Arctic amplification dominated by temperature feedbacks in contemporary climate models. Nat. Geosci., 7, 181-184, https://doi.org/10.1038/ngeo2071.

Roe, G. H., N. Feldl, K. C. Armour, Y.-T. Hwang, and D. M. W. Frierson, 2015: The remote impacts of climate feedbacks on regional climate predictability. Nat. Geosci., 8, 135-139, https://doi.org/ 10.1038/ngeo2346.

Rose, B. E. J., K. C. Armour, D. S. Battisti, N. Feldl, and D. D. B. Koll, 2014: The dependence of transient climate sensitivity and radiative feedbacks on the spatial pattern of ocean heat uptake. Geophys. Res. Lett., 41, 1071-1078, https://doi.org/10.1002/ 2013 GL058955.

_ T. W. Cronin, and C. M. Bitz, 2017: Ice caps and ice belts: The effects of obliquity on ice-albedo feedback. Astrophys. J., 846, 28, https://doi.org/10.3847/1538-4357/aa8306.

Russotto, R. D., and T. P. Ackerman, 2018: Energy transport, polar amplification, and ITCZ shifts in the GeoMIP G1 ensemble.
Atmos. Chem. Phys., 18, 2287-2305, https://doi.org/10.5194/ acp-18-2287-2018.

Sellers, W. D., 1969: A global climatic model based on the energy balance of the Earth-atmosphere system. J. Appl. Meteor., 8 , 392-400, https://doi.org/10.1175/1520-0450(1969)008<0392: AGCMBO $>2.0 . \mathrm{CO} ; 2$.

Shaw, T. A., and A. Voigt, 2016: What can moist thermodynamics tell us about circulation shifts in response to uniform warming? Geophys. Res. Lett., 43, 4566-4575, https://doi.org/ 10.1002/2016GL068712.

Solomon, A., 2006: Impact of latent heat release on polar climate. Geophys. Res. Lett., 33, L07716, https://doi.org/10.1029/ 2005 GL025607.

Viale, F., and T. M. Merlis, 2017: Variations in tropical cyclone frequency response to solar and $\mathrm{CO}_{2}$ forcing in aquaplanet simulations. J. Adv. Model. Earth Syst., 9, 4-18, https://doi.org/ 10.1002/2016MS000785.

Wagner, T. J. W., and I. Eisenman, 2015: How climate model complexity influences sea ice stability. J. Climate, 28, 39984014, https://doi.org/10.1175/JCLI-D-14-00654.1.

Winton, M., 2006: Amplified Arctic climate change: What does surface albedo feedback have to do with it? Geophys. Res. Lett., 33, L03701, https://doi.org/10.1029/2005GL025244.

Zelinka, M. D., and D. L. Hartmann, 2012: Climate feedbacks and their implications for poleward energy flux changes in a warming climate. J. Climate, 25, 608-624, https://doi.org/ 10.1175/JCLI-D-11-00096.1. 